\title{
Further evidence against the classical conditioning model of McCollough effects
}

\author{
D. SKOWBO and T. FORSTER \\ Colby College, Waterville, Maine
}

\begin{abstract}
McCollough effects (MEs) have been portrayed by Murch (1976) as classically conditioned responses which result from the pairing of color (unconditioned stimulus) with line orientation (conditioned stimulus). In the present experiment, we sought evidence that ME characteristics resembled those of undisputed conditioned responses. According to Rescorla (1968) and others, conditioned responses may not develop if the unconditioned stimulus is presented in the absence as well as in the presence of the conditioned stimulus. However, in our experiment, MEs induced by inspection sequences containing presentations of color alone in addition to color/ contour pairs were not weaker than controls. This discrepancy challenges the applicability of the conditioning model to MEs.
\end{abstract}

Perceptual aftereffects whose color depends on the orientation of lines were first reported by McCollough in 1965. After a subject has viewed chromatic lined patterns, achromatic figures of similar spatial configuration may appear to have a desaturated, approximately complementary hue. If, for example, the subject inspects vertical lines on a red background alternating with horizontal lines on a green background for a period of several minutes, subsequently presented achromatic grating patterns may appear greenish on vertical portions and pinkish on horizontal.

One of the most interesting aspects of McCollough effects (MEs) is their longevity. Because the subjective colors can persist for hours or even days, there has been some reservation about models that account for MEs in terms of fatigue or adaptation of neural units. An alternative explanation, formally proposed by Murch in 1976, attributes MEs to classical conditioning. According to this model, the inspection color functions as an unconditioned stimulus (UCS) that produces an adaptive shift in chromatic sensitivity, the unconditioned response. The line configuration paired with color during inspection serves as conditioned stimulus (CS); subsequently, if presented alone, these lines can elicit the adaptive color response. Thus, the tinge of color appearing on achromatic test patterns is regarded as a conditioned response. ${ }^{1}$

One way to evaluate the conditioning model is to determine whether attributes of known conditioned responses are shared by MEs. McCollough effects do have some characteristics that resemble those of con-

This research was supported by funds from Colby College. The authors thank B. Leonard and S. McNamara for their participation. T. Forster is now at Rensselaer Polytechnic Institute, Troy, New York 12180. Requests for reprints should be sent to $D$. Skowbo, Psychology Department, Colby College, Waterville, Maine 04901 . ditioned responses. For example, subjects who view achromatic grating patterns after acquiring MEs lose the effects relatively quickly (Skowbo, Gentry, Timney, \& Morant, 1974); this may be likened to the extinction of a conditioned response. On the other hand, the acquisition of MEs is not facilitated by repeated practice (Skowbo \& Rich, 1982). Clearly, there is a need for further comparisons of MEs with definitive attributes of conditioning or learning.

Kimble (1961) has distinguished between factual and theoretical definitions of learning. While factual definitions relate the phenomena of learning to observable events in the environment, theoretical definitions "describe the essential conditions or the basic processes which the writer believes to be necessary for learning to occur" (p. 2). Most previous comparisons of MEs and conditioned responses seem to have been based on the former type of definition; we felt it would be instructive to attempt a comparison based on the latter.

Rescorla (see, e.g., 1967) has advanced the position that the relevant dimension in associative learning is the contingency between CS and UCS rather than the temporal contiguity between them. This contingency depends on the relative proportion of UCS events that occur in the presence vs. absence of a CS. According to a recent review (Rescorla. \& Holland, 1982), close temporal presentations of CS and UCS will fail to produce conditioning if the UCS is also frequently presented without the CS. The disruptive effect of this maneuver has been shown in several experiments (see, e.g., Gamzu \& Williams, 1971; Leonard, Fischbein, \& Monteau, 1972; Rescorla, 1968).

In the present study, we sought evidence that the interspersion of chromatic stimuli (the UCS in the Murch model) in a series of color/contour pairs (CS + UCS) could hamper the ability of the latter to induce MEs. 


\section{METHOD}

Subjects

Three females and one male participated. Each had colordiscriminating ability in the superior range, as measured by the Farnsworth-Munsell 100-Hue Test; each also had had extensive practice in matching MEs with the color-mixing device described below, having served in previous experiments involving MEs.

\section{Apparatus and Stimuli}

Subjects sat facing a circular piece of acrylic rear-projection material mounted on a black background. Stimuli that appeared on this viewing surface were all 21 deg in diameter.

The stimuli used in inspection sequences included chromatic gratings, homogeneous chromatic stimuli, and a homogeneous achromatic stimulus. The grating patterns were vertical or horizontal green (Wratten No. 53) or magenta (Wratten No. 32) figures; their space-average luminances were, respectively, 22 and $20 \mathrm{~cd} / \mathrm{m}^{2}$, and their spatial frequency was $1.75 \mathrm{c} / \mathrm{deg}$. The homogeneous chromatic stimuli were constructed from the green and magenta filters noted above, and had respective luminances of 47 and $44 \mathrm{~cd} / \mathrm{m}^{2}$. The achromatic homogeneous stimulus, prepared with a neutral-density filter (Wratten No. 96), had a luminance of $52 \mathrm{~cd} / \mathrm{m}^{2}$. The arrangement of these stimuli in inspection sequences is described in the next section.

The test stimulus was a circle with vertical lines on the top half and horizontal lines on the bottom. Overall, its diameter was $21 \mathrm{deg}$, but, in its center, was a 7-deg circular area that was unlined. A homogeneous field of variable chromaticity appeared in this central portion; subjects adjusted it to match MEs appearing on the surrounding lined area. The source of this field was a projection colorimeter that mixed light transmitted by two Wratten color-compensating filters (No. CC30M and No. CC50G). All mixtures could be located in C.I.E. space along a straight line connecting two points with the coordinates $x=.407, y=.454$ and $x=$ $.421, y=.350$. The luminance of this area was $.9 \mathrm{~cd} / \mathrm{m}^{2}$; the spaceaverage luminance of the lined portion was $.64 \mathrm{~cd} / \mathrm{m}^{2}$.

A homogeneous field used for light-adaptation purposes had a luminance of $1.34 \mathrm{~cd} / \mathrm{m}^{2}$.

\section{Inspection Sequences}

Two sets of inspection sequences were prepared; they differed in the proportion of the stimuli that were color/contour pairs. Included within each set were the following conditions:

Set 1. Each inspection sequence in the experimental condition of Set 1 contained 1510 -sec presentations of each chromatic grating stimulus and 15 10-sec presentations of each homogeneous chromatic stimulus. The order of appearance of the stimuli was determined randomly, and, to ensure that no particular role was played by a given order, four different random orders of stimuli were prepared. The sequences lasted $10 \mathrm{~min}$. Of this total, color/ contour pairs were displayed for $5 \mathrm{~min}$; color alone occupied the remaining $5 \mathrm{~min}$.

The control condition contained inspection sequences in which the homogeneous achromatic stimulus was substituted for the homogeneous chromatic stimuli in the experimental condition. The same four random orders of presentation were employed.

In addition, for comparison purposes, there was a standard ME induction sequence in which the two chromatic gratings alternated every $10 \mathrm{sec}$ for $5 \mathrm{~min}$.

Set 2. Inspection sequences in the experimental condition of Set 2 contained 8 presentations of each chromatic grating stimulus and 22 presentations of each homogeneous chromatic stimulus. Again, four different random orders of these stimuli were prepared. In these sequences, color/contour pairs were displayed for $2 \mathrm{~min} 40 \mathrm{sec}$ out of the 10 -min total.

In the control condition, as above, the achromatic homogeneous stimulus was substituted for the chromatic ones. A standard sequence for comparison purposes contained two chromatic gratings alternating every $10 \mathrm{sec}$ for $2 \min 40 \mathrm{sec}$.

\section{Procedure}

Each session began with $3 \mathrm{~min}$ of light adaptation. The subjects were then presented with the test stimulus, after which they gave one match to each half of the pattern. An inspection sequence followed, after which $1 \mathrm{~min}$ of light adaptation was provided. Finally, the test pattern reappeared, and the subjects again matched the two halves.

Two subjects participated in Set 1 conditions only; the other two served in both sets. There were 12 sessions per set-one for each inspection sequence order in the experimental and control conditions plus four standard inspection sequences. Several days elapsed between sessions. Color-orientation combinations were reversed in successive sessions, and preinspection matches to the test pattern assured that no residual effects were present from session to session.

\section{RESULTS}

Each match was converted to C.I.E. coordinates. A simple index of ME strength was taken to be the distance in chromaticity space between loci corresponding to the appearances of the two halves of the test pattern. The mean of these indexes for the four sessions at each condition was obtained for each subject.

Figure 1 shows these means for the experimental and control conditions of Sets 1 and 2. For all participants, these means were higher (i.e., the ME stronger) in the experimental than in the control conditions. Some session-to-session variability was evident, but there were no systematic differences associated with the different sequence orders. Comparisons of the medians of the four sessions at each condition revealed the same pattern of results as obtained using means.

Figure 1 also reveals individual differences among the subjects, with one participant showing consistently lower strength assessments in both sets of data. Data from the two subjects who participated in both sets indicated that strength indexes were lower, over-

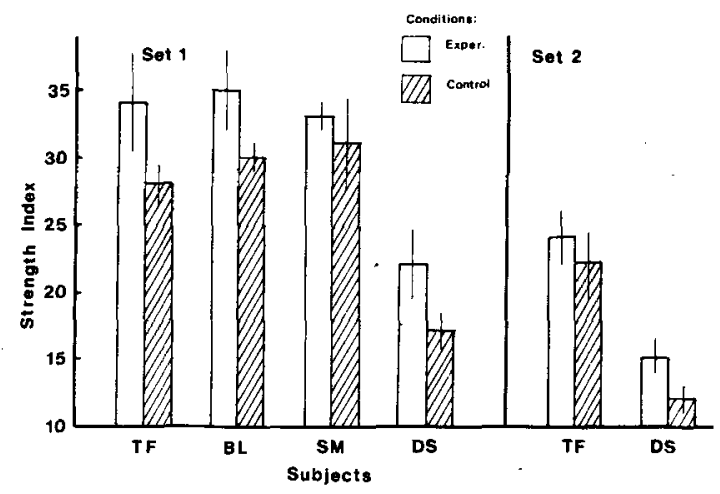

Figure 1. Assessed ME strength following various inspection sequences. Units on the ordinate are distances in C.I.E. space $X$ 103. The abseisen shows experimental and control conditions for each subject in the two sets of inspection sequences. The beight of the bars is a mean of four sessions. Vertical lines show \pm 1 estimated stundard error from these means. 
all, in Set 2; this is consistent with past work showing the relationship between duration of exposure to chromatic gratings and assessed ME strength (see, e.g., Riggs, White, \& Eimas, 1974).

Data from the standard inspection sequences are not shown. The mean of these sessions was always lower than that of the experimental sessions; it was often, but not always, somewhat higher than the mean of the control sessions.

\section{DISCUSSION}

The duration of exposure to chromatic gratings was the same in all conditions within a set. However, the probability of a color occurring in association with a grating was 1.0 in the control conditions, but only .5 (Set 1) or .27 (Set 2) in experimental conditions. Therefore, the contingency between what Murch has called CS and UCS in his (1976) model was substantially reduced in the experimental conditions. Similar circumstances have been shown by Rescorla and others not to produce conditioning. If MEs were conditioned responses, then they should not have appeared in the experimental conditions.

However, our results revealed no decrement in the strength of MEs induced by sequences which contained presentations of unlined chromatic stimuli in addition to chromatic gratings. We take this as evidence against the conditioning model of MEs. Our finding that adding chromatic stimulation to the inspection sequence tended to result in stronger assessed MEs was unexpected, but may be supportive of models featuring sequential processing of color and contour rather than adaptation in units selective for both features.

\section{REFERENCES}

Gamzu, E., \& Williams, D. Classical conditioning of a complex skeletal response. Science, 1971, 171, 923-925.

Kimble, G. Hilgard and Marquis' Conditioning and learning (2nd ed.). New York: Appleton-Century-Crofts, 1961.

Leonard, D., Fischeein, L., \& Monteau, J. The effects of interpolated US alone (USa) presentations on classical nictitating membrane conditioning in rabbit. Conditional Reflex, 1972, $7,107-114$.

McCollough, C. Color adaptation of edge detectors in the human visual system. Science, 1965, 149, $1115-1116$.

Murch, G. Classical conditioning of the McCollough effect: Temporal parameters. Vision Research, 1976, 16, 615-619.

Rescorla, R. Pavlovian conditioning and its proper control procedures. Psychological Review, 1967, 74, 71-80.

Rescorla, R. Probability of shock in the presence and absence of CS in fear conditioning. Journal of Comparative and Physiological Psychology, 1968, 66, 1-5.

Rescorla, R., \& Holland, P. Behavioral studies of associative learning. Annual Review of Psychology, 1982, 83, 265-308.

Riggs, L. A., White, K., \& Eimas, P. Establishment and decay of orientation-contingent aftereffects of color. Perception \& Psychophysics, 1974, 16, 535-542.

Skowbo, D., Gentry, T., Timney, B., \& Morant, R. The McCollough effect: Influence of several kinds of visual stimulation on decay rate. Perception \& Psychophysics, 1974, 16, 47-49.

Skowbo, D., \& Rich, J. Practice does not facilitate acquisition of McCollough effects: Evidence against a learning model. Perception \& Psychophysics, 1982, 32, 551-554.

\section{NOTE}

1. Although there are discrepancies between Murch's model and the traditional classical conditioning paradigm, frequent references to the model appear in ME literature. Therefore, we feel that empirical tests of Murch's proposal are appropriate, and we have assumed the schematics he outlined in order to evaluate it.

(Manuscript received June 27, 1983; revision accepted for publication August 31, 1983.) 